\title{
Um Limitante Superior para o Número Geodésico nos Grafos de Kneser
}

\author{
João V. S. Leite ${ }^{1}$, Marcos Bedo ${ }^{1}$ e Rodolfo A. de Oliveira ${ }^{1}$ \\ ${ }^{1}$ Instituto do Noroeste Fluminense - INFES/UFF \\ Av. João Jasbick, S/N - S. A. Pádua/RJ - Brasil. \\ \{joaovitorleite, marcosbedo, rodolfooliveira\}@id.uff.br
}

\begin{abstract}
This paper provides a preliminary discussion on the geodetic number of Kneser graphs. A set $W, W \subseteq V(G)$, for a graph $G$, is said to be geodetically convex if any vertex in a shortest path between $u$ and $v$ is in $W, \forall u, v \in W$. Such a vertex detection process is called the geodetic interval of $W$, denoted $I[W]$, whereas the geodetic number of $G$ is the smallest cardinality of $W \subseteq V(G)$ such that $I[W]=V(G)$. While finding the geodetic number is known to be an NP-hard problem for generic graphs, in this paper, we examine an upper bound for the geodetic number of Kneser graphs.
\end{abstract}

Resumo. Esse artigo apresenta uma discussão preliminar sobre o número geodésico nos grafos de Kneser. Dado um grafo $G$, um conjunto $W, W \subseteq V(G)$, é dito geodesicamente convexo se qualquer vértice em algum caminho mínimo entre u e v está em $W, \forall u, v \in W$. O processo de detecção desses vértices é chamado de intervalo geodésico de $W$, denotado por $I[W]$, e o número geodésico de $G$ é o menor tamanho de $W \subseteq V(G)$ tal que $I[W]=V(G)$. Embora o problema de encontrar o número geodésico é reconhecidamente NP-difícil para grafos genéricos, mostramos que existe uma função que limita o número geodésico em grafos de Kneser.

\section{Introdução}

O uso de estruturas convexas ganhou notoriedade com o crescimento de problemas envolvendo programação linear em espaços euclidianos [Berger 1990]. Posteriormente, com o intuito de estender o conceito de convexidade para outras estruturas matemáticas além de espaços euclidianos, a convexidade sobre um conjunto $X$ foi formalmente definida como uma coleção $\mathcal{C}$ de subconjuntos de $X$ que satisfazem os três seguintes axiomas: $(i) \emptyset, X \in \mathcal{C} ;(i i) \mathcal{C}$ é fechado por interseção; e $($ iii $) \mathcal{C}$ é fechado por união aninhada [Van de Vel 1993]. De acordo com estes axiomas, os teoremas clássicos de Helly [Helly 1923], Carathéodory [Carathéodory 1911] e Radon [Radon 1921] podem ser unificados em aspectos geométricos comuns nas mais diversas estruturas.

Trabalhos clássicos de convexidade em grafos incluem os estudos de [Farber e Jamison 1986, Duchet 1988, Changat e Mathew 1999] complementados nos trabalhos recentes de [Cáceres et al. 2008, Mathew e Mathew 2018, Marcilon e Sampaio 2018, Dourado et al. 2019]. Em particular, os resultados em [Pelayo 2013] reúnem propriedades importantes de convexidade em grafos, com maior foco na convexidade geodésica. Seguindo os resultados e indicações anteriores, esse trabalho tem seu foco tanto sobre a convexidade geodésica quanto sobre o número geodésico em grafos de Kneser. 


\section{Definições Básicas}

Considere $G=(V, E)$ um grafo finito. O grau de um vértice $v$ corresponde ao número de arestas a ele incidentes em $G$. Um caminho é uma sequência de vértices distintos $x_{1}, x_{2}, \ldots, x_{\ell}$ de $G$ tal que existem as arestas $x_{i}, x_{i+1} \in E$, para $1 \leq i \leq \ell-1$. O comprimento de um caminho é igual ao número de vértices do caminho menos uma unidade e o caminho mínimo é um caminho com menor comprimento entre os vértices extremos. Assim, dado dois vértices $u, v \in V$, a distância entre $u$ e $v$, denotada por $\operatorname{dist}(u, v)$, é o comprimento do caminho mínimo entre $u$ e $v$ no grafo. O diâmetro de $G$, $\operatorname{diam}(G)$, é o maior comprimento dentre todos os caminhos mínimos em $G$.

Um caminho uv-geodésico é um caminho mínimo entre os vértices $u$ e $v$ em $G$. O intervalo geodésico $I[u, v]$ é o conjunto de todos os vértices pertencentes a algum caminho $u v$-geodésico. Para um conjunto $W, W \subseteq V(G)$, o intervalo geodésico $I[W]$ de $W$ é a união dos intervalos geodésicos $I[u, v]$ para todos os pares $u, v \in W$. O conjunto $W$ é chamado de geodesicamente convexo ou $g$-convexo se $I[W]=W$ e é chamado de conjunto geodésico se $I[W]=V(G)$. O número geodésico, $g n(G)$, é a menor cardinalidade para um conjunto geodésico de $G$ e o problema de se encontrar o número geodésico de um grafo é NP-difícil [Dourado et al. 2010].

Dados $n$ e $k$ inteiros positivos, definimos $[2 n+k]=\{1,2, \ldots, 2 n+k\}$ e $[2 n+k]^{n}$ como todos os subconjuntos de $[2 n+k]$ com tamanho $n$. Um grafo de Kneser $K_{n}^{2 n+k}$ é um grafo cujos vértices são formados por $[2 n+k]^{n}$ e cujas arestas existem entre pares de vértices com interseção vazia [Lovász 1978]. Uma característica interessante sobre os grafos de Kneser é que possuem $\left(\begin{array}{c}2 n+k \\ n\end{array}\right)$ vértices cujos respectivos graus equivalem a $\left(\begin{array}{c}n+k \\ n\end{array}\right)$. Na Figura 1 apresentamos o grafo $K_{2}^{5}$ que também é isomorfo ao grafo de Petersen.

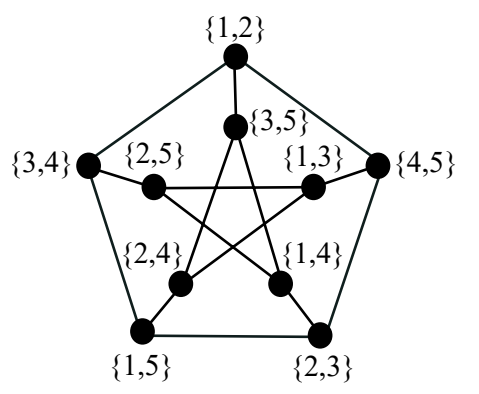

Figura 1. $\mathrm{O}$ grafo de Kneser $K_{2}^{5}$.

Por [Valencia-Pabon e Vera 2005], temos que $\operatorname{diam}\left(K_{n}^{2 n+k}\right)=\lceil(n-1) / k\rceil+1$ e, além disso, para todo $u, v \in V\left(K_{n}^{2 n+k}\right)$ e $|u \cap v|=s$ temos também que:

$$
\operatorname{dist}(u, v)=\left\{\begin{array}{cl}
\min \{2\lceil(n-s) / k\rceil, 2\lceil s / k\rceil+1\} & , \text { para } 1 \leq k<n-1 \\
2 & , \text { se } k \geq n-1
\end{array}\right.
$$

Observe que $2\lceil(n-s) / k\rceil$ corresponde a distância par entre vértices, enquanto que $2\lceil s / k\rceil+1$ a distância impar, para qualquer valor de $s$.

Embora exista muito interesse nos problemas clássicos em teoria dos grafos, como coloração e suas variações envolvendo produtos diretos de grafos de Kneser 
[Brešar e Valencia-Pabon 2019, Balogh et al. 2019, Jin et al. 2020], pouco se conhece sobre resultados de convexidade nessa classe de grafos. Com o objetivo de suprir essa lacuna, esse trabalho investiga, para dado um grafo $K_{n}^{2 n+k}$, uma forma de representar $g n\left(K_{n}^{2 n+k}\right)$ em função de $n$ e $k$.

\section{Resultados}

Primeiramente, apresentamos alguns aspectos que garantem propriedades sobre a distância máxima entre pares de vértices em grafos de Kneser:

Lema 3.1 Sejam $u, v \in V\left(K_{n}^{2 n+k}\right)$. Para $1 \leq k<n-1$, temos que $\operatorname{dist}(u, v)=$ $\operatorname{diam}\left(K_{n}^{2 n+k}\right)$ se, e somente se, $|u \cap v|=s$, para

$$
\left\lceil\frac{n-1}{2 k}\right\rceil \cdot k-k+1 \leq s \leq\left\lceil\frac{n-1}{2 k}\right\rceil \cdot k-k+1+H(n, k)
$$

onde

$$
H(n, k)=\left\{\begin{array}{cl}
\max \{n \bmod k+k-2,0\} & , \text { para } 0 \leq n \bmod k \leq 1 \\
n \bmod k-2 & , \text { para } 2 \leq n \bmod k \leq k-1
\end{array}\right.
$$

Um fato acerca do Lema 3.1 é que interseções inferiores a $\left\lceil\frac{n-1}{2 k}\right\rceil \cdot k-k+1$ fornecem distâncias ímpares, pois podemos reparar que apenas a função à direita produz o mínimo na demonstração da volta do lema, inclusive para os casos $k \geq n-1$. Analogamente, valores maiores que $\left\lceil\frac{n-1}{2 k}\right\rceil \cdot k-k+1+H(n, k)$ geram distâncias pares. Portanto, temos o seguinte resultado:

Corolário 3.1 Sejam $K_{n}^{2 n+k}$ um grafo de Kneser e $u, v \in V\left(K_{n}^{2 n+k}\right)$, então:

- $|u \cap v|<\left\lceil\frac{n-1}{2 k}\right\rceil \cdot k-k+1$ implica dist $(u, v)$ impar; $e$

- $|u \cap v|>\left\lceil\frac{n-1}{2 k}\right\rceil \cdot k-k+1+H(n, k)$ implica $\operatorname{dist}(u, v)$ par.

Agora, vejamos o seguinte resultado:

Teorema 3.1 Sejam $K_{n}^{2 n+k}$ um grafo de Kneser, $r \in V\left(K_{n}^{2 n+k}\right)$ e D o conjunto de todos os vértices de $K_{n}^{2 n+k}$ cuja distância a r seja igual a diam $\left(K_{n}^{2 n+k}\right)$. Então $D \cup\{r\}$ é um conjunto geodésico.

Portanto, com base no Teorema 3.1 e considerando $p=\left\lceil\frac{n-1}{2 k}\right\rceil \cdot k-k+1$, podemos verificar que:

$$
g n\left(K_{n}^{2 n+k}\right) \leq 1+\sum_{i=p}^{s+H(n, k)}\left(\begin{array}{l}
n \\
p
\end{array}\right) \cdot\left(\begin{array}{l}
n+k \\
n-p
\end{array}\right)
$$

onde a primeira parte da soma corresponde ao vértice $r$ e a segunda à cardinalidade de $D$.

\section{Considerações Finais}

A discussão apresentada nesse trabalho permite limitar o comportamento do número geodésico em grafos de Kneser, um resultado preliminar para guiar o estudo e análise da fórmula fechada para o número geodésico em grafos de Kneser. 


\section{Referências}

Balogh, J., Cherkashin, D., e Kiselev, S. (2019). Coloring general kneser graphs and hypergraphs via high-discrepancy hypergraphs. Euro. J. Combinatorics, 79:228 - 236.

Berger, M. (1990). Convexity. Amer. Math. Monthly, 97(8):650-701.

Brešar, B. e Valencia-Pabon, M. (2019). Independence number of products of kneser graphs. Discrete Mathematics, 342(4):1017 - 1027.

Cáceres, J., A.Márquez, e Puertas, M. (2008). Steiner distance and convexity in graphs. Eur. J. Comb., 29(3):726-736.

Carathéodory, C. (1911). Über den variabilitätsbereich der fourierschen konstanten von positiven harmonischen funktionen. Rend. Circ. Mat. Palermo, 32:193-217.

Changat, M. e Mathew, J. (1999). On triangle path convexity in graphs. Discrete Mathematics, 206(1):91-95.

Dourado, M. C., Penso, L. D., e Rautenbach, D. (2019). The hull number in the convexity of induced paths of order 3. In Colbourn, C. J., Grossi, R., e Pisanti, N., editors, Combinatorial Algorithms, pages 214-228, Cham. Springer International Publishing.

Dourado, M. C., Protti, F., Rautenbach, D., e Szwarcfiter, J. L. (2010). Some remarks on the geodetic number of a graph. Discrete Mathematics, 310(4):832 - 837.

Duchet, P. (1988). Convex sets in graphs, II. minimal path convexity. Journal of Combinatorial Theory, Series B, 44(3):307 - 316.

Farber, M. e Jamison, R. E. (1986). Convexity in graphs and hypergraphs. SIAM Journal on Algebraic Discrete Methods, 7(3):433-444.

Helly, E. (1923). Ueber mengen konvexer koerper mit gemeinschaftlichen punkter, jahresber. Math. Verein., 32:175-176.

Jin, Z., Wang, F., Wang, H., e Lv, B. (2020). Rainbow triangles in edge-colored kneser graphs. Applied Mathematics and Computation, 365:124724.

Lovász, L. (1978). Kneser's conjecture, chromatic number, and homotopy. Journal of Combinatorial Theory, Series A, 25(3):319 - 324.

Marcilon, T. e Sampaio, R. (2018). The maximum infection time of the P3 convexity in graphs with bounded maximum degree. Disc. Math., 251:245 - 257.

Mathew, J. K. e Mathew, S. (2018). Monophonic convexity in weighted graphs. Discrete Math., Alg. and Appl., 10(1):1-10.

Pelayo, I. M. (2013). Geodesic Convexity in Graphs. Springer-Verlag New York.

Radon, J. (1921). Mengen konvexer körper, die einen gemeinsamen punkt enthalten. Mathematische Annalen, 83(1-2):113-115.

Valencia-Pabon, M. e Vera, J.-C. (2005). On the diameter of kneser graphs. Discrete Math., 305(1):383 - 385.

Van de Vel, M. (1993). Theory of Convex Structures. North Holland, Amsterdam. 